\title{
Individuals with Mild Intellectual Disability or Borderline Intellectual Functioning in a Forensic Addiction Treatment Center: Prevalence and Clinical Characteristics
}

\author{
Ilse Luteijn ${ }^{1,2}\left(D_{0} \cdot\right.$ Robert Didden $^{3} \cdot$ Joanneke Van der Nagel $^{4}$
}

Published online: 7 July 2017

(C) The Author(s) 2017. This article is an open access publication

\begin{abstract}
Knowledge regarding substance-related problems and offending behavior in individuals with mild intellectual disability or borderline intellectual functioning (MBID; IQ 50-85) has increased over the last years, but is still limited. The present study examined differences in prevalence and clinical characteristics of individuals with and without MBID in a forensic addiction treatment center. Participants were 190 court mandated male clients of a low to high security forensic addiction treatment center in the Netherlands (aged between 21 and 59 years old, $82 \%$ of Dutch origin). Of the total sample $39 \%$ could be identified with MBID which is much higher than the estimated $12 \%$ to $15 \%$ of the general population. Results showed that clients with MBID reported significantly lower scores on desire for help, compared to clients without MBID $(\mathrm{F}(1,73)=5.12, \mathrm{p}=.027)$. Against expectations, no significant group differences were found for aggression during treatment while controlling for impulsivity, treatment duration and type of substance use and offense. As results of the present study showed that clients with MBID are overrepresented in the forensic addiction treatment center,
\end{abstract}

Electronic supplementary material The online version of this article (doi:10.1007/s41252-017-0031-7) contains supplementary material, which is available to authorized users.

Ilse Luteijn

i.luteijn@Tactus.nl

1 Tactus Verslavingszorg, P.O. Box 154, 7400 AD Deventer, the Netherlands

2 Behavioral Science Institute, Radboud University Nijmegen, P.O. Box 9104, 6500 HE Nijmegen, the Netherlands

3 Trajectum, Hanzeallee 2, $8017 \mathrm{KZ}$ Zwolle, the Netherlands

4 Radboud Universiteit Nijmegen / ACSW, NISPA, Postbus 6909, 6503 GK Nijmegen, the Netherlands future research should further explore characteristics and responses to treatment of these clients. More knowledge about the characteristics of clients with MBID will not only help to better identify these clients, but will also to improve treatment for this group

Keywords Mild intellectual disability · Substance use · Offending behavior

\section{Introduction}

It is generally acknowledged that substance use and offending behavior are closely intertwined (Kopak et al. 2014; Vaughn et al. 2016). An estimated 50 to $66 \%$ of offenders meet DSMIV criteria (American Psychiatric Association 2000) for substance abuse or dependence, whereas only $9 \%$ of the general population meet these criteria (Mumola and Karberg 2006). In a sample of clients in forensic mental health services, $78 \%$ met the criteria for at least one lifetime substance use disorder diagnosis (Ogloff et al. 2015). The association between substance use and offending behavior can be explained by several reasons including that substance use may exacerbate psychological symptoms (Swanson et al. 2008), thereby increasing the likelihood of offending behavior (Giancola 2004). Furthermore, offenders were arrested for drug trafficking and/or committed their offense to support their substance use (Kopak et al. 2014). As a result, addiction treatment centers and criminal justice systems often deal with the same individuals and higher percentages of substance abuse and dependency are found in forensic samples (Chandler et al. 2009; Lindsay et al. 2013).

When it comes to substance-related problems and offending behavior, individuals with mild intellectual disability or borderline intellectual functioning (MBID; intelligence quotient (IQ) 50-85) (American Psychiatric Association 
2013) are perceived as a risk group (see Carroll Chapman and Wu 2012; Duijvenbode et al. 2015). Individuals with MBID comprise a substantial part of the population (12-15\%) (Emerson et al. 2010) and experience serious challenges in society (Emerson 2011; Turygin et al. 2014). Besides a greater risk of exposure to social disadvantage, these individuals are 1.2 times more likely to use drugs (Gigi et al. 2014). Several factors like inadequate coping skills (Hartley and MacLean 2008), impaired inhibition (Bexkens et al. 2014), and limited social skills (Carroll Chapman and $\mathrm{Wu} 2012$ ) increase the risk of substance-related problems in individuals with MBID (Duijvenbode et al. 2015). Other factors associated with MBID like susceptibility to social pressure (Taggart et al. 2006) and a lack of inability to understand the potentially severe consequences of substance abuse (Chaplin et al. 2011) also increase the risks in these individuals (Duijvenbode et al. 2015; Slayter and Steenrod 2009).

Although substance-related problems in individuals with MBID have gained more attention in the academic and clinical field (see Carroll Chapman and Wu 2012; Duijvenbode et al. 2015), these problems are still understudied and reliable data on prevalence and risk factors are lacking (VanDerNagel et al. 2013). Researchers who studied substance use in individuals with MBID found that substance use by these individuals was related to various problems including offending behavior (Chapman 2012; Gigi et al. 2014). This might also explain why individuals with MBID constitute a growing percentage of offenders within the justice system and are overrepresented in forensic institutions (Hellenbach et al. 2016; Herrington 2009). In a study by Plant et al. (2011), it was found that half of the clients in a forensic institution could be identified with MBID and substance-related problems. Of these clients, 35\% had used substances prior to the offense leading to their admission. Individuals with MBID and substance-related problems seem to be less likely to receive treatment or to remain in addiction treatment (Carroll Chapman and $\mathrm{Wu}$ 2012). This might explain why these individuals often receive treatment when they are already involved in the justice system due to the consequences of their substance use (Chaplin et al. 2011; Cocco and Harper 2002).

Although it is known that individuals with MBID are overrepresented in both addiction treatment centers and forensic institutions (Bhandari et al. 2014), it remains to be determined (1) what the prevalence of MBID in a forensic addiction treatment center is, (2) what characteristics of this subpopulation are, and (3) if these clients differ in response to treatment, compared to clients without MBID. Considering the risk factors of MBID in both areas, it is important to investigate the prevalence and characteristics of clients in forensic addiction treatment centers. In addition, studying differences in behavior that are evident for the success of treatment provides more insight in if and how clients with MBID differ from clients without MBID. As motivation for treatment is one of the most important factors contributing to the success of treatment (Kopak et al. 2016; Stevens et al. 2015), it is important to study whether motivation differs for clients with MBID compared to those without MBID. This also holds true for aggression during treatment, as this behavior is associated with more dropouts from addiction programs with more risk at relapse in substance use and offending behavior as a result (Bali et al. 2006; Liu et al. 2013). Studying motivation and aggression during treatment thus provides information about differences between clients with and without MBID. Such knowledge could help to increase our knowledge about the clinical characteristics and responses to treatment of clients with MBID.

While legal pressures may be sufficient to enroll a client into treatment, personal motivation for treatment is evident when it comes to commitment to change and to maintain recovery (Groshkova 2010). Increasing motivation thus is an important aspect for all clients; however, it is specifically challenging for clients with MBID (Slayter 2010; Carroll Chapman and Wu 2012). That is, these clients are more likely to externalize the cause of their problems and they also might experience their environment as more negative when compared to clients without MBID (Nader-Grosbois and Vieillevoye 2012). Consequently, they might be in denial or are not aware of the severe consequences of their substance use, which makes them not motivated to make changes or to engage with treatment programs (Taggart et al. 2006). To explore this hypothesis, it should be investigated whether clients with MBID in a forensic addiction treatment center are less motivated for their treatment than clients without MBID.

Just like motivation, aggression during treatment has a large impact on the success of treatment (Liu et al. 2013). Research has shown that aggression is associated with both substancerelated problems and offending behavior (Cuomo et al. 2008) and therefore a common problem in both addiction treatment centers and forensic institutions (Jacob and Holmes 2011; Mericle and Havassy 2008). Aggression is defined in different ways and there are different definitions of aggression (Archer and Coyne 2005). The most widely accepted definition is the one proposed by Berkowitz (1993), a goal-directed behavior that has a deliberate intent to harm or injure another object or person. A number of distinctions can be made, for example, the distinction between direct/overt aggression versus indirect/ covert aggression (Brugman et al. 2015; Richardson and Green 2006). Overt or direct aggression is marked with behaviors that are openly hostile such as hitting, pushing, kicking, biting, and hair pulling. Covert or indirect aggression is marked by hostility, irritability, suspicion, lying, and covert feelings of anger (Olson et al. 2013). Several factors are known to increase the likelihood of aggressive behavior. For example, impulsivity is often related to the emotional and instrumental aspects of aggression (Bevilacqua and Goldman 2013; Hatfield and Dula 2014). Impulsivity can be described as actions which are poorly conceived, prematurely expressed, unduly risky, or 
inappropriate to the situation and that often result in undesirable consequences (Dally et al. 2011). Impulsive individuals are more likely to show difficulties in delay to rewards and often make quick decisions due to a lack of self-control and reflection (Winstanley et al. 2006).

Factors like presence of an intellectual disability seem to increase the likelihood of impulsive behavior (Crocker et al. 2007). Due to impulse control difficulties, individuals with MBID may be less likely to control their behavior, which can lead to the expression of irritability and aggression (Dodge and Pettit 2003; Van Nieuwenhuijzen et al. 2006). In addition, they may not have learned alternative coping skills to deal with stressful situations, which can result in impulsive reactions (Hartley and MacLean 2008). Individuals with good communication skills can often describe their feelings of irritability and frustration, but individuals with lower cognitive and social skills may not be able to identify the reason for their reaction (Hurley 2008). Based on the literature, it can be hypothesized that clients with MBID are more likely to show aggression during treatment in a forensic addiction treatment center compared to clients without MBID. As impulsivity is strongly related to aggression, this should be included when studying aggression during treatment in a forensic addiction treatment center. In addition, as frequency of aggression incidents depends on treatment duration and substance use and offending behavior are associated with aggression (Cuomo et al. 2008; Lynne-Landsman et al. 2011), these factors should be controlled for.

The present study examined if individuals with MBID are overrepresented in forensic addiction treatment centers and whether they differ in their motivation and aggressive behavior during treatment from clients without MBID. To investigate this, data was collected from clients in a forensic addiction treatment center located in the Netherlands. At first, the prevalence of clients with MBID was examined. As an estimated 12 to $15 \%$ of the general population have a MBID and previous research shows that these clients are overrepresented in both addiction and forensic treatment centers, it was expected that more than $15 \%$ of the clients in a forensic addiction treatment center can be identified with MBID (IQ 50-85). Second, as previous research shows that individuals with MBID are less likely to show motivation, it was expected that clients with MBID are less likely to report motivation for treatment than clients without MBID. Finally, as research has shown that MBID is related to higher expressions of overt and covert aggression, it was hypothesized that clients with MBID are more likely to report covert and overt aggression than clients without MBID, while controlling for impulsivity, treatment duration, and type of substance use and index offense. In addition, it was expected that for clients with MBID, more aggression incidents are reported by the clinical staff during treatment than for clients without MBID, while controlling for impulsivity, treatment duration, and type of substance use and index offense.

\section{Method}

\section{Participants}

The present study was conducted in a forensic addiction treatment center with low- to high-security wards in the Netherlands. This clinic offers an integrated approach for substance-related problems, offending behavior, and related problems. The start of the treatment is especially focused on the motivation and examining the psychological functioning of the clients, and the end of the treatment is focused on rehabilitation.

Participants were 190 male inclients between 21 and 59 years old ( $M=38.7$ years, $S D=7.96$ years). The majority of participants were from Dutch origin $(n=157,82 \%)$, while the others originated from Surinam/Dutch Antilles $(n=15$, $8 \%)$, Turkey/Morocco $(n=11,6 \%)$, or other Western $(n=5$, $4 \%)$ or non-Western $(n=4,2 \%)$ countries. Participants were all diagnosed with substance dependence, substance abuse, or/ and pathological gambling according to DSM-IV-TR criteria (American Psychiatric Association 2013). In addition, they were all mandated by court to engage in treatment for substance-related problems and offending behavior. The total average duration of treatment was 7.12 months (range 0-25), and of all participants, $44 \%$ completed their treatment.

Participants were divided into two groups (MBID versus no MBID) based on IQ. IQ was assessed using the most recent scores on the Dutch version of the Wechsler Adult Intelligence Scale third (WAIS-III-NL) (Uterwijk 2000) or fourth edition (WAIS-IV-NL) (Benson et al. 2010) in the participants' file. Substance use-related problems were assessed using DSM-IVTR criteria and diagnosed by an independent professional. Participants were supposed to be abstinent from substances and were monitored during treatment using urine controls. All participants were diagnosed with a substance dependency for one or more substances, and offenses were committed under the influence of substances or were related to substance abuse/dependency. The various types of index offenses were based on the categorization used by the Dutch court system (see Appendix).

\section{Procedure}

In the present study, existing data were used which were collected by the clinical staff in the period of January 2011 to January 2015. There were no pre-defined inclusion criteria to participate in the study, except that clients were admitted to the forensic addiction treatment. When a client started treatment, total IQ was determined by an independent professional. For the present study, all the data of the questionnaires on motivation, impulsivity, and aggression were derived from the client's casefile. The self-report questionnaires were completed in a quiet room by clients with a clinical staff member who 
helped them if necessary. The proxy reports were completed by the clinical staff. All questionnaires were completed after a period of abstinence and all participants finished treatment when data were retrieved from the casefiles when participants finished their treatment. This study was approved by the institute's scientific committee. At the start of their treatment, all clients consented to the use of their casefile data for future scientific studies. As this was a retrospective study using casefile data only, no additional consent was sought.

\section{Measurements}

Intelligence IQ score was assessed with the WAIS-III-NL or WAIS-IV-NL (Wechsler 2008). The WAIS-III and the WAISIV are scales to assess the cognitive ability of adolescents and adults in the age of 16 to 90 . The WAIS-III provides scores for Verbal Intelligence Quotient (VIQ) and Performance Intelligence Quotient (PIQ) along with four secondary indices (verbal comprehension, working memory, perceptual organization, and processing speed). The WAIS-IV has ten subtests which make up four index scores: the Verbal Comprehension Index (VCI), the Perceptual Reasoning Index (PRI), the Working Memory Index (WMI), and the Processing Speed Index (PSI). In previous studies, the test-retest reliabilities ranged from 0.88 to 0.89 and the inter-rater coefficients for the WAIS-III were all above 0.80 (Wechsler 1997). For the WAIS-IV, test-retest reliabilities ranged from 0.70 to 0.90 and the inter-rater coefficients were all above 0.90 (Benson et al. 2010; Wechsler 2008). The Total Intelligence Quotient (TIQ) is based on the scores of the four index scores. The WAIS is the most commonly used test to assess intelligence in adults, also in adults with intellectual disability (MacLean et al. 2011). Results of the WAIS collected in the treatment center were no older than 4 years.

Motivation for Treatment The Dutch version of the Motivation for Treatment (MfT) assesses the person's motivation for treatment. The instrument measures if respondents recognize their problems and whether they would like to have treatment (Weert-Van Oene et al. 2002). The MfT consists of a self-report scale for the client and an observation scale (MfTo) (also indicated as proxy report) for the clinical staff. The questionnaire is divided into four subscales: treatment readiness, desire for help, problem recognition in general, and problem recognition specific (for example substance use and offending behavior). Respondents were given 29 statements such as "Going into treatment may be your last chance to solve your drug/alcohol problems" or "This treatment program can really help you," which they could answer on a five-point Likert scale $(1=$ disagree strongly, $5=$ agree strongly). A higher score on a subscale reflects a greater motivation for treatment. The observation scale for the clinical staff consists of the same statements. Research has shown that the MfT is a reliable and valid instrument; however, this was only tested in a sample of individuals with average intellectual functioning (Weert-Van Oene et al. 2002). In the present study, Cronbach's alpha for the entire sample for the self-report was 0.88 , and for the proxy report, Cronbach's alpha was 0.90 . For the self-reports completed by the clients with MBID, Cronbach's alpha was 0.76 . A Cronbach's alpha of 0.80 was found for the proxy report of the clients with MBID. For the clients without MBID, a Cronbach's alpha of 0.81 was found for the self-report scores and a Cronbach's alpha of 0.87 for the proxy report.

Aggression The Buss-Durkee Hostility Inventory-Dutch version (BDHI-D; Lange et al. 2005) was used to measure aggression at the beginning of treatment. The BDHI-D measures both the experience and the expression of aggression in adolescents and adults from 15 years old. Lange et al. (2005) translated the BDHI into Dutch and validated it for use in the Netherlands. The BDHI-D consists of 40 dichotomized (true of false) items and three scales (i.e., overt aggression, covert aggression, and socially desirability). A high score on the overt aggression scale indicates physical and verbal aggression. An example of such an item is "If somebody hits me first, I let him have it." A high score on the covert aggression scale indicates that an individual experiences aggression and can be an indication for psychopathology. An example of a covert aggression item is "I know that people tend to talk about me behind my back." The internal consistency in previous research on the scale for overt aggression was $\alpha=0.79$ and on the scale for covert aggression $\alpha=0.82$ (Lange et al. 2005). Research has shown that the BDHI-D is a reliable and valid instrument (Lange et al. 2005). The present study shows a reliability for overt aggression of $\alpha=0.69$ and for covert aggression of $\alpha=0.77$. For clients with MBID, a Cronbach's alpha 0.72 was found for the overt aggression and 0.75 for the covert aggression. For clients without MBID, a Cronbach's alpha 0.79 was found for the overt aggression and 0.77 for the covert aggression.

In addition to self-report questionnaires, incidents of aggression were recorded by the clinical staff. When such an incident occurred, the clinical staff reported this in the clients' file. Only the frequency of incidents of aggression was recorded, not the severity or the type of aggressive behavior. In this way, the reports only showed that an aggression incident has occurred, not if this aggression was physical or verbal.

Impulsivity The Dutch version of the Barrat Impulsiveness Scale-11 (BIS-11; Patton et al. 1995) is a well-validated selfreport questionnaire to assess impulsiveness (Stanford et al. 2009). The BIS-11 includes 30 items scored on a four-point Likert scale ( $1=$ never, $4=$ always $)$ describing common impulsive or non-impulsive behaviors and preferences. Example questions include "I say things without thinking," "I get easily bored when solving thought problems," and "I change jobs." 
The degree of impulsivity is measured by adding up the scores of each item. Cronbach's alpha was 0.89 in the present study, which is a slightly higher alpha compared to previous studies in which Cronbach's alpha ranged from 0.79 to 0.83 (Patton et al. 1995). Research has shown that the BIS-11 is a reliable and valid instrument (Stanford et al. 2009).

\section{Data Analyses}

At first, it was assessed for how many clients questionnaires on aggression, impulsivity, and motivation for treatment had been collected. Then, data sets were merged using the ID code of the casefiles and divided into two groups based on the total intelligence score (i.e., IQ 50-85 versus IQ > 85). For the same clients, the frequency of aggression incidents was matched using the ID code of the casefiles. To minimize loss of power, the different types of substances were reduced to the substances that were most often used in both groups (alcohol, cannabis, cocaine/speed, and heroine). Cocaine and speed were combined into one category as these substances are both stimulants (Fletcher et al. 2011), and $85 \%$ of the participants in the study were diagnosed with abuse or dependence on both substances. For type of index offenses, only violent offenses were included as covariate as this type of offense is related to aggression (Vassos et al. 2014).

IBM SPSS Statistics (version 21) was used to conduct the statistical analyses. As for only 75 participants data for motivation for treatment were found, an analysis of variance (ANOVA) was performed to explore group differences in the self-reports and proxy reports for motivation. To minimize loss of power, no covariates were included in this analysis. After conducting the ANOVA, correlations between the self-reports and proxy reports were calculated for the two groups.

A multivariate analysis of covariance (MANCOVA) was assessed to explore group differences on aggressive behavior (i.e., overt aggression, covert aggression, and frequency of aggression incidents) while controlling for impulsivity, treatment duration, and type of substance use and offense. After the assumptions for MANCOVA were checked in SPSS, means and standard deviations were calculated. Then, it was determined whether the MANCOVA was statistically significant by looking at the Wilks' lambda. If this result was statistically significant, post hoc tests were used to explore group differences on aggressive behavior.

With G*Power version 3.1.92, a post hoc power analysis was performed for the MANCOVA with two groups (MBID and no MBID), seven predictors (impulsivity, treatment duration, violent index offense, alcohol, cannabis, cocaine/speed, and heroine), and three response variables (overt aggression, covert aggression, and aggression incidents). Based on the sample size of 190 , an $\alpha$ of 0.05 , and a small effect size of 0.1 , a power of 0.80 was found. This means that for the MANCOVA performed in this study, the sample size should be large enough to detect group differences.

\section{Results}

Clients with and without MBID did not differ in age ( $t$ $(188)=1.58, p=0.115)$ and ethnic background $(F(1$, $188)=0.31, p=0.578)$. In addition, no group differences were found for treatment duration $(t(188)=1.33, p=0.184)$ and successful completion of treatment $(t(190)=0.39, p=0.699)$. Participants were sentenced for various offenses (see Table 1), but mostly for offenses against property. The results in Tables 1 and 2 show that groups did not significantly differ in type of index offense and substance/addiction. Table 3 shows that groups did not significantly differ in the reasons for ending the treatment (e.g., successful completion, relapse in substance use, aggression incident).

\section{Prevalence MBID}

The average total IQ of the entire sample was $87.9(\mathrm{SD}=11.3$, range $=61-125$ ), which is significantly lower than the average intelligence score of 100 of the general population $(t$ $(189)=-14.8, p=0.000)$ (Kaufman 2012). Figure 1 shows the normal distribution of IQ scores of the present sample compared to the normal distribution of the general population. For the total sample, a total IQ score of $87.9(\mathrm{SD}=11.3)$ was found. For clients with MBID, a total IQ score of 78.0 ( $\mathrm{SD}=5.8)$, and for clients without MBID, a total IQ score of 94.5 ( $\mathrm{SD}=9.0$ ), was found. Seventy-five participants (39\%) were identified with MBID, defined as having an IQ between 50 and 85 (American Psychiatric Association 2013; Schalock et al. 2010). This percentage is significantly higher than $12-15 \%$, which would be expected based on the distribution of IQ scores of the general population $(z=9.45$, $p=0.000$ ).

\section{Motivation for Treatment}

Results of Mft self-reports and proxy reports were found for 30 clients with MBID and 45 clients without MBID. The results of the one-way ANOVA are presented in Table 4. No significant group differences were found regarding self-reports of readiness for treatment, problem recognition general, and problem recognition specific. However, group differences were found for desire for help $(F(1,73)=2.15, p=0.027)$, with clients with MBID showing significantly lower scores on this variable than clients without ID. This means that clients with MBID are less likely to experience a desire for help at the beginning of treatment compared to clients without MBID. Looking at the proxy reports, no group differences were found on all four motivation treatment scales. This means that the clinical staff did not report any differences between groups on the several measurements for motivation at the beginning of treatment.

To explore the relationships between the different variables, correlations between self-reports and proxy reports of 
Table 1 Types of index offenses $(n=190)$

\begin{tabular}{lllrl}
\hline & MBID $(n=75)$ & $\begin{array}{l}\text { No MBID } \\
(n=115) \\
\%\end{array}$ & $t(188)$ & $p$ value \\
\hline Property offenses & $\%$ & 89.3 & 1.66 & 0.099 \\
Violent offenses & 96.1 & 68.8 & 0.40 & 0.688 \\
Vandalism and public-order offenses & 71.1 & 25.9 & -0.82 & 0.414 \\
Drug-related offenses & 25.0 & 20.5 & 0.41 & 0.682 \\
Traffic violations & 23.7 & 22.3 & -0.83 & 0.410 \\
Possession of weapons & 17.1 & 10.7 & 0.23 & 0.543 \\
Sex offenses & 14.5 & 1.9 & -0.16 & 0.247 \\
\hline
\end{tabular}

Multiple responses were possible. clients with MBID without MBID were calculated (see Table 5 and Fig. 2). For clients with MBID, significant correlations were found between self-reports and proxy reports for desire for help and problem recognition general. For clients without MBID, significant correlations were also found for these scales, and for readiness for treatment. Looking at the correlations and plots, there is a large difference between the self-reports and proxy reports on the readiness for treatment scale. Self-reports and proxy reports were correlated for clients without MBID, but not for clients with MBID.

\section{Aggression}

For 188 participants, aggression measurements could be assessed. The number of aggression incidents per participant ranged from 0 to 3 . Table 6 shows the results of the MANCOVA controlling for impulsivity, treatment duration, and type of substance use and index offense. Of the 192 clients who completed the aggression self-report questionnaires, 188

Table 2 Types of substance use/addiction $(n=190)$

\begin{tabular}{lllll}
\hline & MBID $(n=75)$ & $\begin{array}{l}\text { No MBID } \\
(n=113) \\
\%\end{array}$ & $t(188)$ & $p$ value \\
& $\%$ & 58.0 & 0.68 & 0.497 \\
Alcohol & 61.8 & 56.3 & 1.16 & 0.246 \\
Cannabis & 64.5 & 81.3 & -0.43 & 0.667 \\
Cocaine & 78.9 & 36.6 & -1.02 & 0.308 \\
Heroine & 28.9 & 19.6 & -1.12 & 0.265 \\
Speed & 13.2 & 7.1 & -0.35 & 0.729 \\
GHB & 6.7 & 8.0 & -0.55 & 0.580 \\
XTC & 6.6 & 6.3 & 0.22 & 0.828 \\
Medication & 7.9 & 4.4 & 0.32 & 0.701 \\
Gambling & 4.2 & 1.7 & 0.90 & 0.299 \\
Other & a & 3.1 & &
\end{tabular}

Multiple responses were possible

a The category "other" includes substances that are not very commonly used or new types of drugs such as ketamine, LSD, Qat, and new psychoactive stimulants participants also completed the impulsivity questionnaires at the beginning of their treatment. Results of Wilks' lambda showed that groups did not significantly differ on the levels of aggression, with $F(3,183)=0.138, p=0.937$, Wilk's $\Lambda=0.998$, and partial $\eta^{2}=0.002$. As this result is not statistically significant, no further follow-up tests based on intelligence were performed. Although no significant group differences were found for the aggression measurements, results did show a significant effect of impulsivity on overt $(F(1$, $179)=47.31, p=0.000)$ and covert aggression $(F(1$, $179)=43.01, p=0.000)$, meaning that clients who did report more impulsivity were also more likely to report more overt $(\beta=4.77, t=6.70, p=0.000)$ and covert aggression $(\beta=6.08$, $t=6.59, p=0.000$ ). In addition, a significant effect of violent index offenses was found on frequency of aggression incidents $(F(1,179)=6.79, p=0.010)$, which means that clients who committed a violent offense before treatment were more likely to be reported for an aggression incident during treatment $(\beta=0.23, t=2.64, p=0.010)$.

\section{Discussion}

The present study examined the prevalence and characteristics of clients with mild intellectual disability or borderline intellectual functioning (MBID; IQ 50-85) in a forensic addiction treatment center. In addition, it was investigated whether clients with MBID differed in motivation and aggression during treatment compared to clients without MBID.

The first hypothesis concerned the higher prevalence rate of clients with MBID in a forensic addiction treatment center as $39 \%$ of the sample could be identified with MBID. This result confirms the hypothesis that more than $15 \%$ of the clients in a forensic addiction treatment center could be identified with MBID, based on the 12 to $15 \%$ found in the general population (Emerson et al. 2010). This result is in line with results based on studies in forensic institutions and prisons showing that 10 to $40 \%$ of the clients could be identified with a MBID (Lindsay et al. 2013; Ståhlberg et al. 2010) and that 
Table 3 Reasons for termination of treatment $(n=190)$

\begin{tabular}{lrrrr}
\hline & MBID $(n=75)$ & $\begin{array}{l}\text { No MBID } \\
n=113) \\
\%\end{array}$ & $t(188)$ & $p$ value \\
\hline Successful completion & $\%$ & 43.2 & 0.42 & 0.678 \\
Aggression & 46.1 & 8.0 & -1.52 & 0.129 \\
Relapse in substance use & 2.6 & 19.6 & 0.07 & 0.941 \\
Relapse in delinquent behavior & 19.7 & 0.9 & -0.82 & 0.416 \\
Leaving without permission & 0.0 & 28.6 & 0.13 & 0.899 \\
Other $^{\text {a }}$ & 26.3 & 8.9 & 0.96 & 0.377 \\
\hline
\end{tabular}

Multiple responses were possible

a The category "other" includes reasons like not following the rules of the treatment center and transfer to another center where treatment was more suitable high percentages of these clients reported substance-related problems (Bhandari et al. 2014). The results of the present study show that clients with MBID are also overrepresented in forensic addiction treatment centers. This result can be explained by a range of factors that make individuals MBID more at risk for substance-related problems and offending behavior, such as inadequate coping skills (Hartley and MacLean 2008), susceptibility to social pressure (Taggart et al. 2006), and lack of inability to understand the severe consequences of substance use (Chaplin et al. 2011). In addition, previous research showed that individuals with MBID are less likely to receive treatment or to remain in voluntary treatment, compared to clients without MBID (Carroll Chapman and Wu 2012). This might also explain the higher prevalence rates of clients with MBID in the forensic addiction treatment center.

The second hypothesis concerned differences between clients with and without MBID in motivation for treatment with the expectation that clients with MBID were less motivated for treatment than clients without MBID. At first, differences in self-reports were investigated. In line with our expectation, results showed that clients with MBID reported lower scores on desire for help than clients without MBID. This result can be explained by the fact that individuals with MBID are more likely to externalize the cause of their problems and therefore may be less likely to admit that they need help (NaderGrosbois and Vieillevoye 2012; Taggart et al. 2006). In addition, they may not fully understand the severe consequences of their problems and therefore have the feeling that they can solve their problems on their own (Chaplin et al. 2011).

Although a significant difference was found for desire for help, no group differences were found on the other self-report scales. Also on the proxy reports, no significant group differences were found. These results are not in line with our expectations that clients with MBID are more likely to be in denial of their substance-related problems and therefore less motivated to make changes regarding their substance use (Taggart et al. 2006). An explanation is that the subsample that completed the motivation for treatment questionnaires was too small to detect any group differences. Another explanation is the interpretation of the motivation in clients with MBID by the clinical staff. Correlations in the present study showed that self-reports and proxy reports for readiness for treatment were significantly correlated for clients without MBID but not for clients with MBID. The clinical staff reported high scores on readiness for treatment for clients with MBID, which may indicate that the staff overestimates these clients on this specific variable or underestimate problems in these clients. This result is also interesting as it is known that there are methodological and measurement issues while studying behavior in clients with MBID (Carroll Chapman and $\mathrm{Wu}$ 2012). The reliability of self-reports completed by clients with MBID can be affected by their limited cognitive
Fig. 1 Histogram of the distribution of IQ scores in the present sample compared to the theoretical sample (general population)

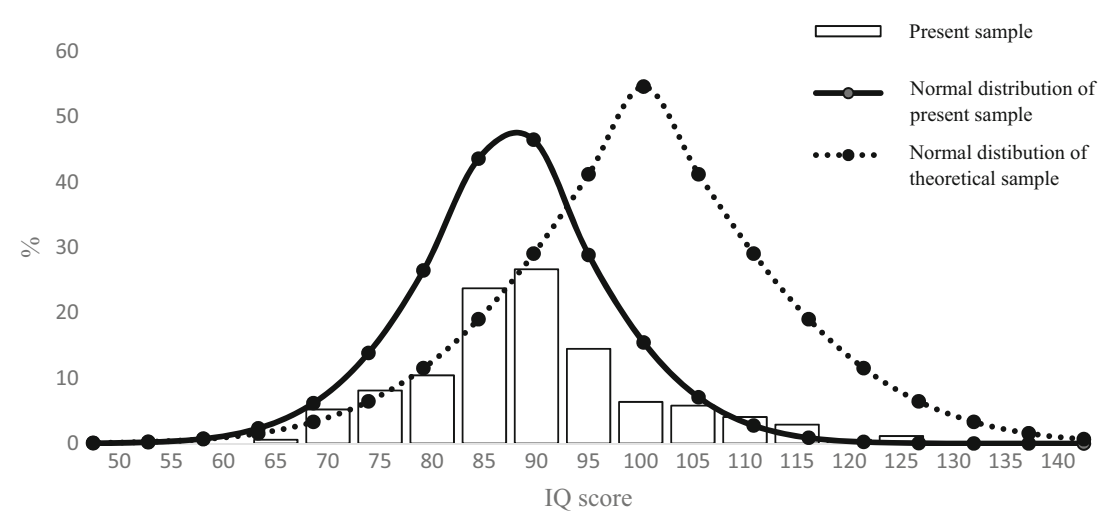


Table 4 Results of a one-way analysis of variance for group differences on motivation for treatment $(n=75)$

\begin{tabular}{|c|c|c|c|c|c|c|}
\hline & \multicolumn{2}{|c|}{$\operatorname{MBID}(n=30)$} & \multicolumn{2}{|c|}{ No MBID $(n=45)$} & \multirow[t]{2}{*}{$F(1,73)$} & \multirow[t]{2}{*}{$p$ value } \\
\hline & $M$ & SD & $M$ & SD & & \\
\hline \multicolumn{7}{|l|}{ Self-report } \\
\hline Readiness for treatment & 3.97 & 0.51 & 3.98 & 0.59 & 0.00 & 0.990 \\
\hline Desire for help & 3.48 & 0.65 & 3.82 & 0.65 & 5.12 & 0.027 \\
\hline Problem recognition general & 3.61 & 1.24 & 4.00 & 0.93 & 2.36 & 0.129 \\
\hline Problem recognition specific & 3.22 & 1.21 & 3.54 & 0.93 & 1.67 & 0.200 \\
\hline \multicolumn{7}{|l|}{ Proxy report } \\
\hline Readiness for treatment & 3.75 & 0.53 & 3.57 & 0.49 & 1.19 & 0.171 \\
\hline Desire for help & 3.59 & 0.61 & 3.57 & 0.63 & 0.07 & 0.791 \\
\hline Problem recognition general & 3.85 & 0.86 & 3.70 & 0.84 & 0.48 & 0.491 \\
\hline Problem recognition specific & 3.56 & 0.85 & 3.48 & 0.73 & 0.169 & 0.682 \\
\hline
\end{tabular}

$M$ mean, $S D$ standard deviation

capacities, verbal comprehension, and self-reflection (Schalock et al. 2010). In addition, it is known that clients with MBID are also more susceptible for desirable answers (Jobson et al. 2013; Langdon et al. 2010). Furthermore, proxy reports such as those of clinical staff members often underestimate clients' substance-related problems (Wilson et al. 2004). This indicates that we have to be careful with the interpretation of measurements in clients with MBID in both the academic and clinical field. More research on the application of self-reports and proxy reports has important implications for the treatment of clients with MBID.

The last hypothesis concerned group differences in aggression during treatment while controlling for impulsivity, treatment duration, and type of substance use and index offense. In contrast with our expectations, results showed that clients with MBID did not report more overt and covert aggression during treatment than clients without MBID. In addition, no group differences were found for the frequency of aggression incidents during treatment, controlled for impulsivity, treatment duration, and type of substance use and offense. Although results could not be compared with previous research, several studies show that clients with MBID are more likely to show aggressive behavior due to impulse control difficulties and inadequate coping skills (Hartley and MacLean 2008; van Nieuwenhuijzen et al. 2006). Just like for the motivation for

Table 5 Correlations between self-reports and proxy reports of motivation for treatment scales $(n=75)$

\begin{tabular}{lcl}
\hline & MIBD & No MBID \\
\hline Readiness for treatment & 0.16 & $0.52^{* *}$ \\
Desire for help & $0.41^{*}$ & $0.37^{*}$ \\
Problem recognition general & $56^{* *}$ & $0.39^{* *}$ \\
Problem recognition specific & 0.20 & 0.20 \\
\hline
\end{tabular}

$* p<0.05, * * p<0.001$ treatment questionnaires, it could be that the self-report questionnaires for aggression were not fully adapted for clients with MBID. Especially as reliability for both overt and covert aggression in the present study was under 0.80 , this should be taken in consideration when interpreting the results. In addition, the records of aggression incidents in the present study may not be specific enough and sensitive to the interpretation of the clinical staff which is also indicated by other studies (Vereenooghe and Langdon 2013). However, in contrast to previous research, it could also be that clients with MBID did not differ in their aggression compared to those without MBID. For these reasons, measuring and interpreting aggression behavior in clients with MBID should be examined in future research.

\section{Strengths, Limitations, and Future Directions}

This study has several strengths. Most important, the results of the present study provided more knowledge about the prevalence and clinical characteristics of clients with MBID in a forensic addiction treatment center compared to those without MBID. As such, it provides important insights for both the academic and clinical field, for example, that clients with MBID are overrepresented (i.e., 39\%) in the forensic addiction treatment center. This study can be the beginning of future research on the characteristics and differences in response to treatment in clients with MBID in forensic addiction treatment centers. Finally, the present study indicated the importance of using suitable and reliable self-reports and proxy reports in clients and stresses the need for more research on this topic. Especially looking at the large number of clients with MBID in the forensic addiction treatment center, it is important to examine which instruments are used, how these are interpreted, and what this means for the treatment of these clients.

The results of this study should be interpreted in the context of a number of shortcomings. As this was a clinic-based study 
Fig. 2 Plots of the correlations between self-reports and proxy reports for the motivation for treatment scales $(n=75)$
MBID

No MBID

Readiness for treatment

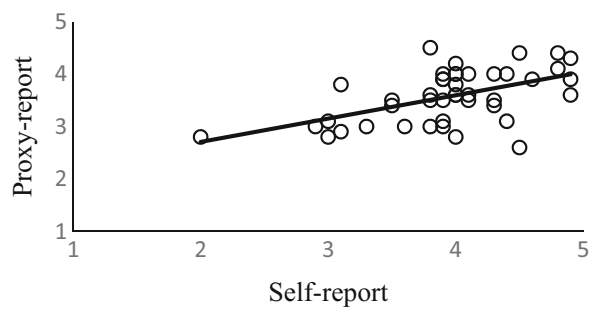

Desire for help
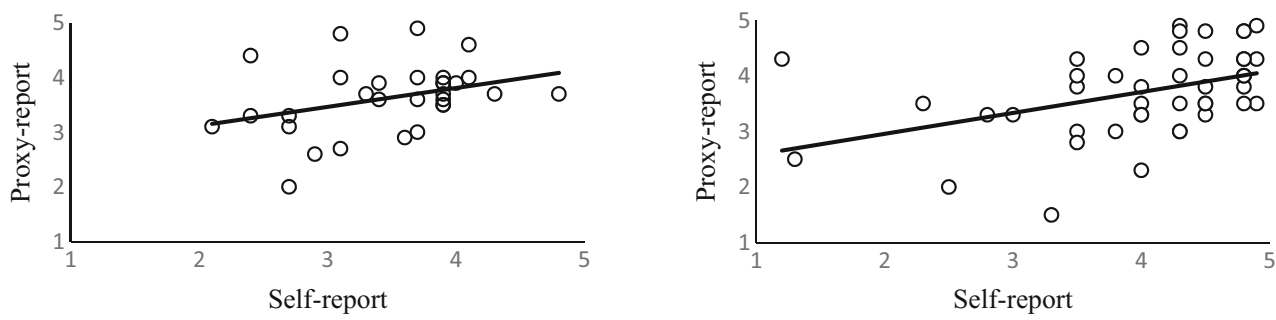

Problem recognition general
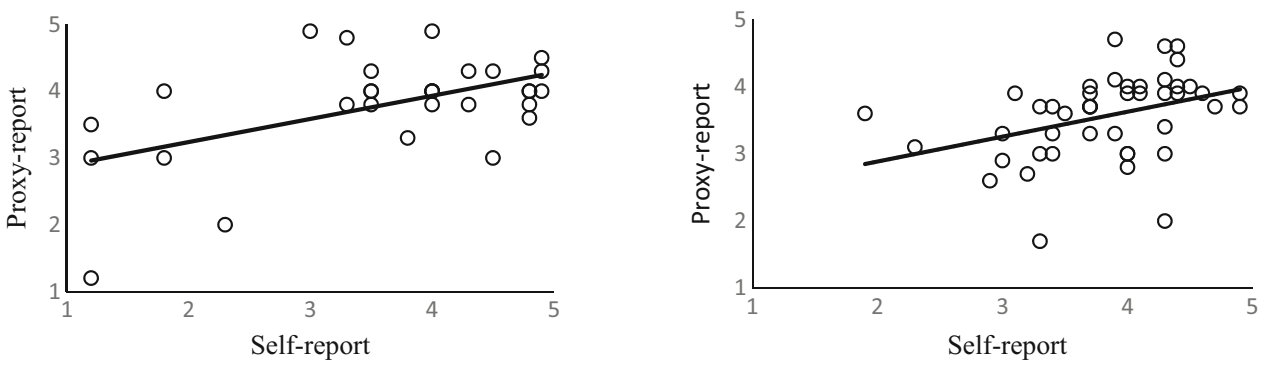

Problem recognition specific

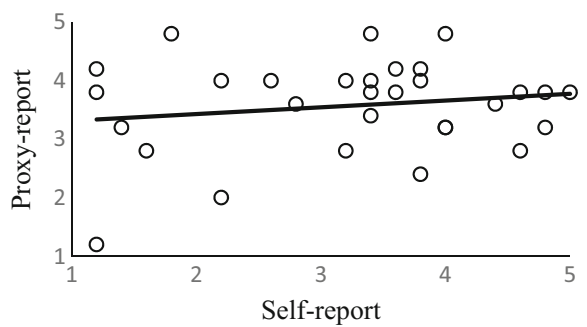

using existing data, this has several consequences for the interpretation of the results. At first, only first measurements of

Table 6 Group differences on aggression measurements $(n=188)$

\begin{tabular}{|c|c|c|c|c|c|c|}
\hline & \multicolumn{2}{|l|}{ MBID } & \multicolumn{2}{|c|}{ No MBID } & \multirow[t]{2}{*}{$F(1,179)$} & \multirow[t]{2}{*}{$p$ value } \\
\hline & $M$ & SD & $M$ & SD & & \\
\hline Overt aggression & 12.42 & 0.39 & 12.31 & 0.48 & 0.07 & 0.793 \\
\hline Covert aggression & 10.75 & 0.05 & 9.97 & 0.62 & 0.80 & 0.371 \\
\hline Aggression incidents & 0.27 & 0.05 & 0.29 & 0.06 & 0.06 & 0.814 \\
\hline
\end{tabular}

motivation for treatment and aggression self-reports could be included, which means that differences in the course of the treatment process could not be examined. In addition, only a small subsample for the motivation for treatment questionnaires could be included, which negatively influences the power of the analysis. Furthermore, IQ scores were derived from the results of the WAIS-III and WAIS-IV. The question is how valid these results are when measuring MBID in combination with a past of substance abuse. In addition, results of the WAIS-III and WAIS-IV are not fully comparable and some measurements were older than 2 years. This also applies to the measurements of motivation for treatment, impulsivity, and aggression. 
Finally, the study was performed in one single-treatment center and included only male clients, which means that the results cannot be generalized to other institutions and samples.

Several suggestions for future research can be made. As results of the present study showed that clients with MBID are indeed overrepresented in the forensic addiction treatment center, future research should further investigate the treatment of substance-related problems and offending behavior in this group. Next to that, it should be further examined how differences in motivation and aggression differ during entire treatment using repeated measures. Especially for differences in aggression incidents, it would be interesting to include the time until the incident occurs using Poisson regression survival analysis (VanDeSande et al. 2011). Other factors should also be included like differences in motives for substance use (Thornton et al. 2012), coping skills (Hartley and MacLean 2008; van Nieuwenhuijzen et al. 2006), and executive functioning (Willner et al. 2010). Furthermore, differences in selfreports and proxy reports should be further investigated. Especially as results in the present study indicate that selfreports and proxy reports for readiness for treatment were more correlated for clients without MBID, it should also be studied whether instruments in forensic addiction treatment centers are appropriate for and fully adapted to clients with MBID. Especially as underrecognition of problems in clients with MBID is a risk (Wieland and Zitman 2016), this should be taken in consideration when interpreting the results of the proxy reports completed by the clinical staff. As this might influence the treatment of clients with MBID, it is important to investigate whether this is the case. Furthermore, it would be useful to investigate whether clients with MBID are more likely to relapse in substance use and/or offending behavior during and after treatment performing a follow-up study. Especially as there are few specialized treatments available for this specific group, it should be studied how this influences the success of treatment and recidivism of clients with MBID. When more knowledge about the clinical characteristics and risk factors in these clients is available, treatment can be improved.

The present study showed that of the clients in a forensic addiction treatment center, $39 \%$ could be identified with MBID. In addition, it was found that clients with MBID are less likely to experience a desire for help compared to clients without MBID. Future research should further explore differences in motivation between clients with and without MBID and how these instruments are interpreted by the clients and the clinical staff. Clients with MBID form a specific group in the forensic addiction treatment centers which requires specialized treatment from multidisciplinary teams with knowledge of substance-related problems, offending behavior, and MBID. More research is necessary to adapt these specialized treatments to the needs and intellectual functioning of clients with MBID and eventually prevent them for relapsing in substance use and offending behavior.

Acknowledgements This research was financed by Tactus Verslavingszorg. The authors are grateful to all clients and clinical staff who participated in the study.

Author Contributions IL: designed and executed the study, analyzed the data, and wrote concepts of the paper. RD: advised on the design, assisted with data analyses and writing of the paper. JVDN: advised on the design, assisted with the data analyses and writing of the paper.

\section{References}

American Psychiatric Association (2000). Diagnostic and statistical manual of mental disorders (4th ed.). Washington, DC: APA.

American Psychiatric Association (2013). Diagnostic and statistical manual of mental disorders (5th ed.). Washington, DC: APA.

Archer, J., \& Coyne, S. (2005). An integrated review of indirect, relational, and social aggression. Personality and Social Psychology Review, 9, 212-230. doi:10.1207/s15327957.

Bali, S. A., Carroll, K. M., Canning-Ball, M., \& Rounsaville, B. J. (2006). Reasons for dropout from drug abuse treatment: symptoms, personality, and motivation. Addictive Behaviors, 31, 320-330. doi:10. 1016/j.addbeh.2005.05.013.

Benson, N., Hulac, D. M., \& Kranzler, J. H. (2010). Independent examination of the Wechsler Adult Intelligence Scale-fourth edition (WAIS-IV): what does the WAIS-IV measure? Psychological Assessment, 22, 121-130. doi:10.1037/a0017767.

Berkowitz, L. (1993). Aggression: its causes, consequences and control. New York: McGraw-Hill.

Bevilacqua, L., \& Goldman, D. (2013). Genetics of impulsive behaviour. Philosophical Transactions of the Royal Society, 368, 1-4. doi:10. 1098/rstb.2012.0380.

Bexkens, A., Ruzzano, L., Collot d'Escury-Koenigs, A. M. L., Van der Molen, M. W., \& Huizenga, H. M. (2014). Inhibition deficits in individuals with intellectual disability: a meta-regression analysis. Journal of Intellectual Disability Research, 58, 3-16. doi:10.1111/ jir.12068.

Bhandari, A., van Dooren, K., Eastgate, G., Lennox, N., \& Kinner, S. A. (2014). Comparison of social circumstances, substance use and substance-related harm in soon-to-be released prisoners with and without intellectual disability. Journal of Intellectual Disability Research, 59, 571-579. doi:10.1111/jir.12162.

Brugman, S., Lobbestael, J., Arntz, A., Cima, M., Schuhmann, T., Dambacher, F., \& Sack, A. T. (2015). Identifying cognitive predictors of reactive and proactive aggression. Aggressive Behavior, 41, 51-64. doi:10.1002/ab.21573.

Carroll Chapman, S. L., \& Wu, L. T. (2012). Substance abuse among individuals with intellectual disabilities. Research in Developmental Disabilities, 33, 1147-1156. doi:10.1016/j.ridd.2012.02.009.

Chandler, R. K., Fletcher, B. W., \& Volkow, N. D. (2009). Treating drug abuse and addiction in the criminal justice system: improving public health and safety. The Journal of the American Medical Association, 301, 183-190. doi:10.1001/jama.2008.976.

Chaplin, E., Gilvarr, C., \& Tsakanikos, E. (2011). Recreational substance use patterns and co-morbid psychopathology in adults with intellectual disability. Research in Developmental Disabilities, 32, 29812986. doi:10.1016/j.ridd.2011.05.002.

Cocco, K. M., \& Harper, D. C. (2002). Substance use in people with retardation: a missing link in understanding community outcomes? Rehabilitation Counseling Bulletin, 46, 33-40. doi:10.1177/ 00343552020460010301 . 
Crocker, A. G., Mercier, C., Allaire, J. F., \& Roy, M. E. (2007). Profiles and correlates of aggressive behavior among adults with intellectual disabilities. Journal of Intellectual Disabilities, 51, 786-801.

Cuomo, C., Sarchiapone, M., Giannantonio, M. D., Mancini, M., \& Roy, A. (2008). Aggression, impulsiveness, personality traits, and childhood trauma of prisoners with substance abuse and addiction. The American Journal of Drug and Alcohol Abuse, 34, 339-345. doi:10. 1080/00952990802010884.

Dalley, J. W., Everitt, B. J., \& Robbins, T. W. (2011). Impulsivity, compulsivity, and top-down cognitive control. Neuron, 69, 680-694.

Dodge, K. A., \& Pettit, G. S. (2003). A biopsychosocial model of the development of chronic conduct problems in adolescence. Developmental Psychology, 39, 349-371. doi:10.1037//0012.

Emerson, E. (2011). Health status and health risks of the "hidden majority" of adults with intellectual disability. Intellectual and Developmental Disabilities, 49, 155-165. doi:10.1352/1934-955649.3.155.

Emerson, E., Einfeld, S., \& Stancliffe, R. (2010). The mental health of young children with intellectual disabilities or borderline intellectual functioning. Social Psychiatry and Psychiatric Epidemiology, 45, 579-587. doi:10.1007/s00127-009-0100.

Fletcher, P. J., Rizos, Z., Noble, K., \& Higgins, G. A. (2011). Impulsive action induced by amphetamine, cocaine and MK801 is reduced by 5-HT 2C receptor stimulation and 5-HT 2A receptor blockade. Neuropharmacology, 61, 468-477.

Giancola, P. R. (2004). Executive functioning and alcohol-related aggression. Journal of Abnormal Psychology, 113, 541-555. doi:10.1037/ 0021-843X.113.4.541.

Gigi, K., Werbeloff, N., Goldberg, S., Portuguese, S., Reichenberg, A., Fruchter, E., \& Weiser, M. (2014). Borderline intellectual functioning is associated with poor social functioning, increased rates of psychiatric diagnosis and drug use: a cross sectional population based study. European Neuropsychopharmacology, 24, 17931797. doi:10.1016/j.euroneuro.2014.07.016.

Groshkova, T. (2010). Motivation in substance misuse treatment. Addiction Research \& Theory, 18, 494-510. doi:10.3109/ 16066350903362875.

Hartley, S. L., \& MacLean, W. E. (2008). Coping strategies of adults with mild intellectual disabilities for stressful social interactions. Journal of Mental Health Research in Intellectual Disabilities, 1, 109-127. doi:10.1080/19315860801988426.

Hatfield, J., \& Dula, C. S. (2014). Impulsiveness and physical aggression: examining the moderating role of anxiety. The American Journal of Psychology, 127, 233-243. doi:10.5406/amerjpsyc.127.2.0233.

Hellenbach, M., Karatzias, T., \& Brown, M. (2016). Intellectual disabilities among prisoners: prevalence and mental and physical health comorbidities. Journal of Applied Research in Intellectual Disabilities, 30, 230-241. doi:10.1111/jar.12234.

Herrington, V. (2009). Assessing the prevalence of intellectual disability among young male prisoners. Journal of Intellectual Disability Research, 53, 397-410. doi:10.1111/j.1365-2788.2008.01150.

Hurley, A. D. (2008). Depression in adults with intellectual disability: symptoms and challenging behaviour. Journal of Intellectual Disability Research, 52, 905-916. doi:10.1111/j.1365-2788.2008. 01113.

Jacob, J. D., \& Holmes, D. (2011). Working under treat: fear and nurseclient interactions in forensic psychiatric setting. Journal of Forensic Nursing, 7, 68-77. doi:10.1111/j.1939-3938.2011.01101.

Jobson, L., Stanbury, A., \& Langdon, P. E. (2013). The Self- and OtherDeception Questionnaires-Intellectual Disabilities (SDQ-ID and ODQ-ID): component analysis and reliability. Research in Developmental Disabilities, 34, 3576-3582. doi:10.1016/j.ridd. 2013.07.004.

Kaufman, J. C. (2012). Self-estimates of general, crystallized, and fluid intelligence in an ethnically diverse population. Learning and
Individual Differences, 22, 188-122. doi:10.1016/j.lindif.2011.10. 001.

Kopak, A. M., Vartanian, L., Hoffman, N. G., \& Hunt, D. E. (2014). The connections between substance dependence, offense type, and offense severity. Journal of Drug Issues, 44, 291-307. doi:10.1177/ 0022042613511439 .

Kopak, A. M., Hoffman, N. G., \& Proctor, S. L. (2016). Key risk factors for relapse and rearrest among substance use treatment clients involved in the criminal justice system. American Journal of Criminal Justice, 41, 14-30. doi:10.1007/s12103-015-9330-6.

Langdon, P. E., Clare, I. C. H., \& Murphy, G. H. (2010). Measuring social desirability amongst men with intellectual disabilities: the psychometric properties of the Self- and Other-Deception QuestionnaireIntellectual Disabilities. Research in Developmental Disabilities, 31, 1601-1608.

Lange, A., Hoogendoorn, M., Wiederpahn, A., \& de Beurs, E. (2005). Buss-Durkee hostility inventory -Dutch, BDHI-D. Handleiding, verantwoording en normering van de Nederlandse Buss-Durkee agressievragenlijst [manual, validity and stardards of the DutchBuss-Durkee agression questionnaire]. Houten: Bohn Stafleu van Loghum.

Lindsay, W. R., Carson, D., Holland, A. J., Taylor, J. L., O’Brien, G., Wheeler, J. R., et al. (2013). Alcohol and its relationship to offence variables in a cohort of offenders with intellectual disability. Journal of Intellectual and Developmental Disability, 38, 325-331. doi:10. 3109/13668250.2013.837154.

Liu, J., Lewis, G., \& Evans, L. (2013). Understanding aggressive behavior across the life span. Journal of Psychiatric and Mental Health Nursing, 20, 156-168. doi:10.1111/j.1365-2850.2012.01902.

Lynne-Landsman, S. D., Graber, J. A., Nichols, T. R., \& Botvin, G. J. (2011). Trajectories of aggression, delinquency, and substance use across middle school among urban, minority adolescents. Aggressive Behavior, 37, 161-176.

MacLean, H., McKenzie, K., Kidd, G., Murray, A. L., \& Schwannauer, M. (2011). Measurement invariance in the assessment of people with an intellectual disability. Research in Developmental Disabilities, 32, 1081-1085. doi:10.1016/j.ridd.2011.01.022.

Mericle, A. A., \& Havassy, B. E. (2008). Characteristics of recent violence among entrants to acute mental health and substance abuse services. Social Psychiatry and Psychiatric Epidemiology, 43, 392 402. doi:10.1007/s00127-008-0322-4.

Mumola, C. J., \& Karberg, J. C. (2006). Drug use and dependence, state and federal prisoners, 2004. Washington, DC: US Department of Justice, Office of Justice Programs, Bureau of Justice Statistics.

Nader-Grosbois, N., \& Vieillevoye, S. (2012). Variability of selfregulatory strategies in children with intellectual disability and typically developing children developing children in pretend play situations. Journal of Intellectual Disability Research, 56, 140-156. doi:10.1111/j.1365-2788.2011.01443.

van Nieuwenhuijzen, M., Orobio de Castro, B., Van der Valk, I., Wijnroks, L., Vermeer, A., \& Mattys, W. (2006). Do social information processing models explain aggressive behavior by children with mild intellectual disabilities in residential care? Journal of Intellectual Disability Research, 50, 801-812. doi:10.1111/j.13652788.2005.00773.

Ogloff, J. R. P., Talevski, D., Lemphers, A., Wood, M., \& Simmons, M. (2015). Co-occurring mental illness, substance use disorders, and antisocial personality disorder among clients of forensic mental health services. Psychiatric Rehabilitation Journal, 38, 16-23.

Olson, S. L., Sameroff, A. J., Lansford, J. E., Sexton, H., Davis-Kean, P., Bates, J. E., et al. (2013). Deconstructing the externalizing spectrum: growth patterns of overt aggression, covert aggression, oppositional behavior, impulsivity/inattention, and emotion dysregulation between school entry and early adolescence. Development and Psychopathology, 25, 817-842. doi:10.1017/S0954579413000199. 
Patton, J. H., Stanford, M. S., \& Barrat, E. S. (1995). Factor structure of the Barrat Impulsiveness Scale. Journal of Clinical Psychology, 51, 768-774. doi:10.1002/1097-4679.

Plant, A., McDermott, E., Chester, V., \& Alexander, R. T. (2011). Substance misuse among offenders in a forensic intellectual disability service. Journal of Learning Disabilities and Offending Behaviour, 2, 127-135.

Richardson, D. S., \& Green, L. R. (2006). Direct and indirect aggression: relationships as social context. Journal of Applied Social Psychology, 36, 2492-2508. doi:10.1111/j.0021-9029.2006.00114.

Schalock, R. L., Borthwick-Duffy, S. A., Bradley, V., Buntinx, W. H., Coulter, D. L., Craig, E. M., et al. (2010). Intellectual disability: definition, classification and systems of support (11th ed.). Washington, DC: American Association on Intellectual and Developmental Disabilities (AAIDD).

Slayter, E. M. (2010). Demographic and clinical characteristics of people with intellectual disabilities with and without substance abuse disorders in a Medicaid population. Intellectual and Developmental Disabilities, 48, 417-431.

Slayter, E., \& Steenrod, S. A. (2009). Addressing alcohol and drug addiction among people with mental retardation in nonaddiction settings: A need for cross-system collaboration. Journal of Social Work Practice in the Addictions, 9, 71-90. doi:10.1080/ 15332560802646547.

Ståhlberg, O., Anckarsäter, H., \& Nilsson, T. (2010). Mental health problems in youths committed to juvenile institutions: prevalences and treatment needs. European Child \& Adolescent Psychiatry, 19, 893903. doi:10.1007/s00787-010-0137-1.

Stanford, M. S., Mathias, C. W., Dougherty, D. M., Lake, S. L., Anderson, N. E., \& Patton, J. H. (2009). Fifty years of the Barratt Impulsiveness Scale: An update and review. Personality and individual differences, 47, 385-395.

Stevens, L., Verdejo-García, A., Roeyers, H., Goudriaan, A. E., \& Vanderplasschen, W. (2015). Delay discounting, treatment motivation and treatment retention among substance-dependent individuals attending an in inclient detoxification program. Journal of Substance Abuse Treatment, 49, 58-64.

Swanson, J. W., Van Dorn, R. A., Swartz, M. S., Smith, A., Elbogen, E. B., \& Monahan, J. (2008). Alternative pathways to violence in persons with schizophrenia: the role of childhood antisocial behavior problems. Law and Human Behavior, 32, 228-240. doi:10.1007/ s10979-007-9095-7.

Taggart, L., McLaughlin, D., Quinn, B., \& Milligan, V. (2006). An exploration of substance misuse in people with learning disabilities. Journal of Intellectual Disability Research, 50, 588-597.

Thornton, L. K., Baker, A. L., Lewin, T. J., Kay-Lambkin, F. J., Kavanagh, D., Richmond, R., et al. (2012). Reasons for substance use among people with mental disorders. Addictive Behaviors, 37, 427-434.

Turygin, N., Matson, J. L., \& Adams, H. (2014). Prevalence of cooccurring disorders in a sample of adults with mild and moderate intellectual disabilities who reside in a residential treatment setting. Research in Developmental Disabilities, 35, 1802-1808. doi:10. 1016/j.ridd.2014.01.027.

Uterwijk, J. (2000). WAIS-III Nederlandstalige bewerking. Technische handleiding. Lisse: Swets \& Zeitlinger.

VanDerNagel, J. E. L., Kemna, L. E., \& Didden, R. (2013). Substance use among persons with mild intellectual disability: approaches to screening and interviewing. NADD Bulletin, 16, 87-92.

VanDeSande, R., Nijman, H. L. I., Noorthoorn, E. O., Wierdsma, A. I., Hellendoorn, E., VanDerStaak, C., \& Mulder, C. L. (2011). Aggression and seclusion on acute psychiatric wards: effect of short-term risk assessment. The British Journal of Psychiatry, 199, 473-478.

VanDuijvenbode, N., VanDerNagel, J. E., Didden, R., Engels, R. C. M. E., Buitelaar, J. K., Kiewik, M., \& de Jong, C. A. (2015). Substance use disorders in individuals with mild to borderline intellectual disability: current status and future directions. Research in Developmental Disabilities, 38, 319-328.

Vassos, E., Collier, D. A., \& Fazel, S. (2014). Systematic meta-analyses and field synopsis of genetic association studies of violence and aggression. Molecular Psychiatry, 19, 471-477.

Vaughn, M. G., Salas-Wright, C. P., \& Reingle-Gonzalez, J. M. (2016). Addiction and crime: the importance of asymmetry in offending and the life-course. Journal of Addictive Diseases, 18, 1-5. doi:10.1080/ 10550887.2016.1189658.

Vereenooghe, L., \& Langdon, P. (2013). Psychological therapies for people with intellectual disabilities: a systematic review and meta-analysis. Research in Developmental Disabilities, 34, 4085-4202. doi: 10.1016/j.ridd.2013.08.030.

Wechsler, D. (1997). Wechsler Adult Intelligence Scale for Adults-third edition. San Antonio, TX: The Psychological Corporation.

Wechsler, D. (2008). Wechsler Adult Intelligence Scale-fourth edition: technical and interpretive manual. San Antonio, TX: Pearson.

de Weert-Van Oene, G. H., Schippers, G. M., De Jong, C. A., \& Schrijvers, G. A. (2002). Motivation for treatment in substancedependent clients. Psychometric evaluation of the TCU motivation for treatment scales. European Addiction Research, 8, 2-9.

Wieland, J., \& Zitman, F. G. (2016). It is time to bring borderline intellectual functioning back into the main fold of classification systems. The British Journal of Psychiatry, 40, 204-206.

Willner, P., Bailey, R., Parry, R., \& Dymond, S. (2010). Evaluation of executive functioning in people with intellectual disabilities. Journal of Intellectual Disability Research, 54, 366-379.

Wilson, C. R., Sherritt, L., Gates, E., \& Knight, J. R. (2004). Are clinical impressions of adolescent substance use accurate? Pediatrics, 114, 536-540.

Winstanley, C. A., Eagle, D. M., \& Robbins, T. W. (2006). Behavioral models of impulsiveness in relation to ADHD: translation between clinical and preclinical studies. Clinical Psychology Review, 26, 379-396. doi:10.1016/j.cpr.2006.01.001. 